\title{
Real-Time Characterization of Period-Doubling Dynamics in Uniform and Dispersion Oscillating Fiber Ring Cavities
}

\author{
Florent Bessin, François Copie, Matteo Conforti, Alexandre Kudlinski, and Arnaud Mussot \\ Université Lille, CNRS, UMR 8523-PhLAM-Physique des Lasers Atomes et Molécules, \\ F-59000 Lille, France \\ Stefano Trillo \\ Department of Engineering, University of Ferrara, Via Saragat, 44122 Ferrara, Italy
}

(Received 18 March 2019; revised manuscript received 10 September 2019; published 11 November 2019)

\begin{abstract}
Modulational instability in passive optical resonators, the triggering mechanism of frequency comb and pulse train generation, is shown to exhibit transitions between regimes involving period-one (P1) versus period-two (P2) dynamical evolutions. The latter is a signature of parametric resonance occurring in the system, which can arise either from intrinsic cavity periodicity or from spatial modulation of the cavity parameters. We characterize the P1-P2 transition for both cases, employing a fiber resonator where the intracavity fiber can be either uniform or dispersion modulated. The key element of our setup is a time lens which we exploit to resolve the temporal dynamics over successive round trips, allowing crystal-clear evidence of the existence of P1-P2 transitions for suitable changes of cavity parameters, as well as for the successful characterization of the relative temporal patterns. Our findings reveal new regimes where the averaged model known as the Lugiato-Lefever equation turns out to be inadequate to explain the dynamics, whereas the results are correctly predicted and described on the basis of the full Ikeda map.
\end{abstract}

DOI: 10.1103/PhysRevX.9.041030

Subject Areas: Nonlinear Dynamics, Optics

\section{INTRODUCTION}

The generation of frequency combs has attracted a lot of attention during the last decades since these ultraprecise optical rulers have a wealth of applications including astrophysics, metrology, or spectroscopy to name a few $[1,2]$. Also associated with the formation of cavity solitons in the time domain $[3,4]$, their generating mechanism relies on modulation instability (MI), which first initiates the exponential growth of two symmetric sidebands around the pump [5,6]. Then, subsequent four-wave mixing processes lead to the generation of additional equidistant lines to eventually form ultrabroadband spectra that might reach an octave [7]. Characteristics of the frequency comb thus strongly depend on these early stages of formation, and a perfect knowledge of the MI dynamics is essential to understand their formation in order to optimize their performances [8]. The first observation of MI in passive resonators has been achieved by Coen and Haelterman [9]

\footnotetext{
florent.bessin@univ-lille.fr
}

Published by the American Physical Society under the terms of the Creative Commons Attribution 4.0 International license. Further distribution of this work must maintain attribution to the author(s) and the published article's title, journal citation, and DOI. in an all-fiber system. This seminal work has motivated further investigations aimed at understanding the complex and rich dynamics of MI processes that may arise beyond the basic configuration. More recent investigations have addressed passive fiber ring cavities in the weak dispersion regime [10-12], understrong cavity driving to reach nonlinear shifts larger than $2 \pi$ [7,13-15], through polarization effects [16,17], and in dispersion-modulated cavities [18-21].

The common thread between these complex systems, compared to the basic configuration studied in Refs. [6,9], is that new parametric resonances are excited [7,10-16,18-22]. This striking feature is not only limited to the generation of new sets of frequencies but also to the modification of the whole behavior of the system. From a theoretical point of view, these new parametric resonances correspond to new eigenvalues of the system, which might be positive or negative, thus leading to temporal shifts of the output pattern from round trip to round trip $[14,20,23,24]$. In modulationaly unstable systems [5,25], the output cavity pulse train can either be out-of-phase round trip to the other, which is denoted as the P2 regime or, conversely, in phase in the socalled $\mathrm{P} 1$ regime [20]. In particular, the $\mathrm{P} 2$ regime turns out to be inherently associated with the parametric resonance phenomenon induced by system periodicity. However, different sources of periodicity such as the intrinsic periodicity associated with the cavity boundary conditions in a 
uniform cavity, or ad hoc introduced modulation of the cavity parameters (e.g., dispersion, nonlinearity, etc.), can have different impacts on how the parametric resonance reflects itself into the onset of $\mathrm{P} 2$ regimes. To date, however, only indirect evidence of such phenomena have been reported through observation of spectral sideband generation (see Ref. [26] for a uniform cavity and Refs. [19,27] for a cavity with modulation of dispersion). Conversely, the true assessment of $\mathrm{P} 2$ regimes by means of real-time observations over the timescale of the round trip remains extremely challenging and has not been reported so far. The general importance of such types of measurements stands also on the fact that the P2 regime is a well-known mechanism in nonlinear systems supporting bistable states, first predicted by Ikeda et al. [28], which was identified as a first step in a universal route to chaos. It has been observed in modulationaly stable passive fiber cavities [29-31] and investigated in active lasers in theory [32-34] and in experiments [35,36].

In this paper, we report direct observations in the time domain of $\mathrm{P} 1$ and $\mathrm{P} 2$ regimes in both uniform and modulated passive fiber ring cavities. We implemented a time lens [37], which is a recent instrumentation developed to study optical rogue waves in real time $[38,39]$ or to characterize transient dynamics in active lasers [40]. This device based on space-time duality [41] temporally magnifies the cavity output pulse train whose period lies in the picosecond scale to reach nanosecond duration that can be measured in real-time, round trip to round trip, using high-speed photodetectors. Thus, $\pi$ or $2 \pi$ shifts experienced by the output pulse train round trip to round trip can be observed and thus the nature of the regime, P1 or P2, identified. Moreover, we provide theoretical development to support these experimental observations.

The paper is organized as follows. In Sec. II, a theoretical description of both uniform (Sec. II B) and modulated cavities (Sec. II C) is presented. We end up with parametric gain expressions that allow us to describe the dynamics and to identify the P1 and P2 regimes. In Sec. III, experimental results are presented. First, the experimental setup used in both cavity configurations is described (Sec. III A), followed by experimental results in a uniform passive fiber cavity (Sec. III B 1) and a dispersion-modulated one (Sec. III B 2).

\section{THEORETICAL INVESTIGATIONS}

\section{A. Governing equations}

We consider a passive fiber ring cavity modeled by the following Ikeda map model [28]:

$$
\begin{aligned}
& \frac{\partial E_{n}(z, t)}{\partial z}=\left(-\frac{\alpha_{f}}{2}-i \frac{\beta_{2}(z)}{2} \frac{\partial^{2}}{\partial t^{2}}+i \gamma\left|E_{n}(z, t)\right|^{2}\right) E_{n}(z, t), \\
& E_{n+1}(0, t)=\rho E_{n}(L, t) \exp \left(i \Phi_{0}\right)+\theta E_{\text {in }}(t),
\end{aligned}
$$

where Eq. (1) is the nonlinear Schrödinger equation (NLSE) that rules the propagation of the intracavity field $E_{n}$ (respectively, intracavity power $P=\left|E_{n}\right|^{2}$ ) during the $n$th round trip along the cavity of length $L$, in the reference frame moving at the wave group velocity. The parameters $\beta_{2}, \gamma$, and $\alpha_{f}$ are, respectively, the group velocity dispersion (GVD), the nonlinear coefficient, and the fiber loss coefficient. Equation (2) accounts for the periodic boundary conditions imposed by the coupler at each round trip, where $\rho$ and $\theta$ are, respectively, the reflection and transmission coefficients of the coupler defined such that $\rho^{2}+\theta^{2}=1$. The parameter $E_{\text {in }}$ refers to the pump input field (respectively, input power $P_{\text {in }}=\left|E_{\text {in }}\right|^{2}$ ), and $\Phi_{0}$ is the linear phase accumulated by the intracavity field over one round trip. The cavity detuning $\delta_{0}$ is defined such as $\delta_{0}=2 k \pi-\Phi_{0}$, with $k$ an integer, chosen such that the cavity detuning refers to the detuning of the pump frequency from the closest resonance of the cavity. Note that while it is often very convenient to investigate the dynamics of this system using a simplified model such as the Lugiato-Lefever equation (LLE) [5], this model is not valid for large values of the cavity detuning $[10,13,15,20]$. Some of the results presented here were obtained outside the range of validity of this model, which is thus not fully relevant for our study.

In the following, we first study the emergence of the P1 and $\mathrm{P} 2$ regimes by means of Floquet theory in the most simple cavity configuration: the uniform fiber cavity. Then, we extend this analysis to the dispersion of a modulated fiber cavity.

\section{B. Uniform fiber cavities}

A schematic of the passive cavity is shown in Fig. 1(a) along with the evolution of the GVD over one cavity round trip in Fig. 1(b). As detailed in Ref. [20], the dynamics of the system is well described by performing a linear stability analysis of the complete map system [Eqs. (1) and (2)] using the method described in Refs. [9,42]. We obtain a system of differential equations whose evolution is ruled by the product of the fundamental matrix calculated from the linear stability analysis of Eq. (1) (at $z=L$ ) and the rotation matrix owing to the boundary conditions [Eq. (2)]. As a result, the stability of this system depends on eigenvalues of the product of these two matrices. For the modulus of eigenvalues larger than one, the steady-state cw solution is unstable with respect to harmonic perturbation, which initially grows exponentially. Assuming that fiber propagation losses are small relative to coupling losses, the overall cavity losses can be approximated by $\alpha=$ $1-\rho \exp \left(-\alpha_{f} / L\right)$, which leads to eigenvalues expressed as [20]

$$
\lambda_{ \pm}=(1-\alpha)\left[\Psi \pm \sqrt{\Psi^{2}-1}\right],
$$

where 

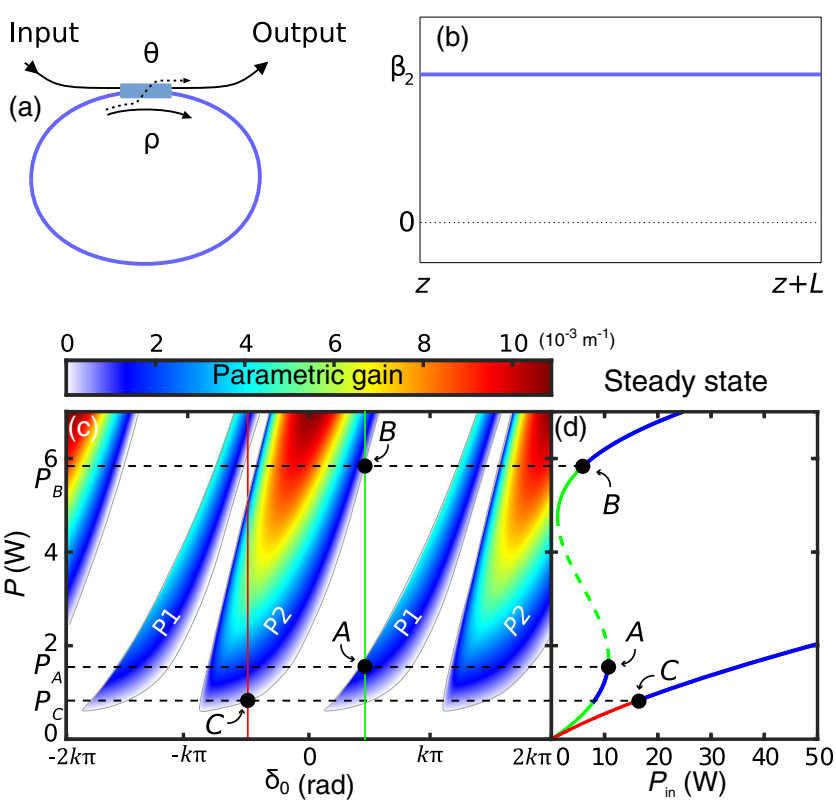

FIG. 1. (a) Schematic of the cavity. (b) GVD evolution over one cavity round trip. (c) Two-dimensional (2D) map of the maximum parametric gain in the plan $\left(\delta_{0}, P\right)$. (d) Steady-state curves of the cavity for $\delta_{0}=1.44 \mathrm{rad}$ (green curve) and $\delta_{0}=-1.6 \mathrm{rad}$ (red curve). The blue parts stand for the regions where the P1 and P2 regimes can be excited. Parameters are as follows: $\beta_{2}^{\mathrm{DSF}}=9 \mathrm{ps}^{2} \cdot \mathrm{km}^{-1}, L=120.6 \mathrm{~m}, \gamma=2.5 \mathrm{~W}^{-1} \cdot \mathrm{km}^{-1}$, and cavity losses $\alpha=0.165$.

$$
\Psi=[\cos (\mu L) \cos (\Phi)-\kappa \sin (\mu L) \sin (\Phi)]
$$

The parameter $\Phi=\gamma L P+\Phi_{0}$ refers to the total phase accumulated over a round trip, while

$$
\mu=\sqrt{\frac{\beta_{2}^{2} \Omega^{4}}{4}+\beta_{2} \gamma P \Omega^{2}}
$$

refers to the standard MI gain, with $\Omega$ the pulsation of the perturbation and $\kappa=\left(\beta_{2} \Omega^{2} / 2+\gamma P\right) / \mu$. It can easily be checked that unstable eigenvalues (i.e., $|\lambda|>1$ ) appear only for $|\Psi|>[1-\alpha+1 /(1-\alpha)] / 2$, which leads to real eigenvalues that can be expressed as $\lambda=|\lambda| e^{i m \pi}$, with $m$ an integer. As a consequence, an infinite number of frequencies can be destabilized, each of them being associated with a parametric resonance of the system. We introduce $\lambda^{\prime}$, which corresponds to the eigenvalue $\lambda_{ \pm}$with the highest modulus; hence, the complex gain turns out to be $\ln \left(\left|\lambda^{\prime}\right|\right) / L+i m \pi / L$. Two distinct cases appear depending on the value of $m$ : (i) For $m$ even (i.e., $\lambda^{\prime}>1$ ), the initial perturbation grows exponentially [with growth rate $g_{\text {uniform }}=\ln \left(\left|\lambda^{\prime}\right|\right) / L$ ] following P1 dynamics. (ii) For $m$ odd (i.e., $\lambda^{\prime}<-1$ ), the perturbation changes sign at every round trip, which is characteristic of P2 dynamics. Clear-cut evidence of this behavior is the $\pi$ phase shift experienced by the temporal pattern from round trip to round trip as opposed to the P1 case. An overview of the behavior of the system in terms of P1 or P2 regimes can be obtained by looking at the maximum gain value calculated from the growth rate $g_{\text {uniform }}$ in the $\left(\delta_{0}, P\right)$ plane [see Fig. 1(c)]. This representation is relevant since it allows a comprehensible description of the dynamics of the systems, and these parameters correspond to those easily accessible from an experimental point of view. In this work, we focus our attention on the case of normal dispersion, which more easily reveals the striking features of the system. Indeed, cavities operating in the anomalous dispersion regime are known to exhibit MI under a rather large range of parameters, which is likely to hinder the observation of a competition between P1 and P2 dynamics. Figure 1(c) shows that the system exhibits an alternation of narrow and broad instability tongues associated with P1 and P2 dynamics, respectively. As soon as the pump power is above a certain threshold that depends on the amount of losses, the system might enter one of the unstable regions. We can point out that this mapping is necessarily $2 \pi$ periodic with respect to $\delta_{0}$, owing to the periodicity of the linear resonances of the cavity. Accordingly, the most noteworthy features of the system can be illustrated by considering only two scenarios at the MI cavity threshold: the first one, where the nonlinear phase is close to zero, and the second one, where the phase is close to $\pi$. These two cases have been called resonant and antiresonant cases in Ref. [9], respectively. Nevertheless, well above the MI threshold, this denomination is not relevant anymore. Indeed, the nonlinear phase shift $\left(\Phi_{\mathrm{NL}}=\gamma L P\right)$ induces a displacement of resonance peaks of several radians; thus, in this paper, we use the denomination positive and negative detuning $\left(\delta_{0}\right)$ in order to correctly identify the different scenarios.

First, let us consider the cavity operating on positive detuning. As an example, we set the cavity detuning to $\delta_{0}=1.44 \mathrm{rad}$, indicated by the vertical green line in Fig. 1(c). For this value of detuning, the system is bistable, and two distinct instability tongues can be accessed, depending on the intracavity power. For a better understanding, we plot in Fig. 1(d) the corresponding steadystate curve function:

$$
P=\frac{\theta^{2} P_{\text {in }}}{1+(1-\alpha)^{2}-2(1-\alpha) \cos (\Phi)},
$$

in the $\left(P_{\text {in }}, P\right)$ plane (green curve). The dashed line between the knees of this $S$-shaped curve delimits the "unconditionably unstable domain" regarding the continuous wave (cw), while the blue lines indicate unstable states in regards to unstable domains in Fig. 1(c). With a pump power above the threshold for the first instability tongue on the lower branch of the steady-state curve $\left[P_{A}=1.56 \mathrm{~W}\right.$, point $A$ in Figs. 1(c) and 1(d)], a pulse train that is stable from one round trip to the other can be excited and thus corresponds to P1 dynamics. This lower branch is known to be 
modulationaly unstable if $\Delta>4.25$, with $\Delta=\delta_{0} / \alpha$, and it is linked to the so-called Turing instability $[43,44]$. By increasing the intracavity power to $P_{B}=5.84 \mathrm{~W}$ [point $B$ in Figs. 1(c) and 1(d)], the system switches to the upper branch of the steady-state curve [see Fig. 1(d)] and falls within another parametric instability tongue that is associated with P2 dynamics. We point out that such a behavior could not be anticipated using the Lugiato-Lefever model, which predicts a steady state for the system $[18,25,43]$.

In the second scenario, the cavity detuning is set to a negative value. For example, $\delta_{0}=-1.6 \mathrm{rad}$ [vertical red line in Fig. 1(c)]. The steady-state curve for this detuning is plotted in Fig. 1(d) (red curve), and we notice that the P2 instability can be accessed for a lower intracavity power $\left[P_{C}=0.83 \mathrm{~W}\right.$, point $C$ in Figs. $1(\mathrm{c})$ and $\left.1(\mathrm{~d})\right]$. At this large negative detuning value, it is also possible to switch on the P1 tongue with a huge increase of pump power to $P_{\text {in }} \approx 180 \mathrm{~W}$, which is not accessible with standard experimental equipments.

\section{Dispersion-modulated fiber cavities (DMF)}

A similar mathematical approach can be applied to modulated cavities [20]. For the sake of simplicity, we focus on the specific case where only the GVD is modulated along the cavity length with a piecewise constant dispersion profile, corresponding to almost all realistic fiber optics configurations [45]. However, a similar behavior is expected to be observed when varying any other cavity parameter regardless of the exact modulation format $[22,46]$. A schematic of the cavity is shown in Fig. 2(a) along with the GVD evolution $\beta_{2}(z)$ [Fig. 2(b)]. Here, we consider a cavity built out of two pieces of uniform fibers of lengths $L_{a}$ and $L_{b}$ with GVD coefficients $\beta_{2, a}$ and $\beta_{2, b}$, respectively. This case corresponds to the simplest configuration for which the period of dispersion $\left(\Lambda=L_{a}+L_{b}\right)$ is equal to the cavity length $L$. We define the ratio between these lengths $N=(L / \Lambda)$; thus, $N=1$ in this example. This value can be arbitrarily larger, and for the sake of generality, theoretical investigations have been performed for any integer values of $N$. As in the previously studied case of uniform cavities, we investigate the dynamics of this system thanks to a linear stability analysis of the complete map system [Eqs. (1) and (2)]. We obtain a system of difference equations whose evolution is ruled by the product of the fundamental matrices of each uniform fiber calculated from Eq. (1) and a rotation matrix owing to the boundary conditions (2). The stability of this system depends on eigenvalues of the product of these matrices, which can be expressed in the form of Eq. (3) over a period $\Lambda$, where now

$$
\begin{aligned}
\Psi= & {\left[\cos \left(\mu_{a} L_{a}\right) \cos \left(\mu_{b} L_{b}\right)-\sigma_{1} \sin \left(\mu_{a} L_{a}\right) \sin \left(\mu_{b} L_{b}\right)\right] } \\
& \times \cos (\Phi)-\sin (\Phi) \\
& \times\left[\sigma_{2} \sin \left(\mu_{a} L_{a}\right) \cos \left(\mu_{b} L_{b}\right)+\sigma_{3} \cos \left(\mu_{a} L_{a}\right) \sin \left(\mu_{b} L_{b}\right)\right],
\end{aligned}
$$
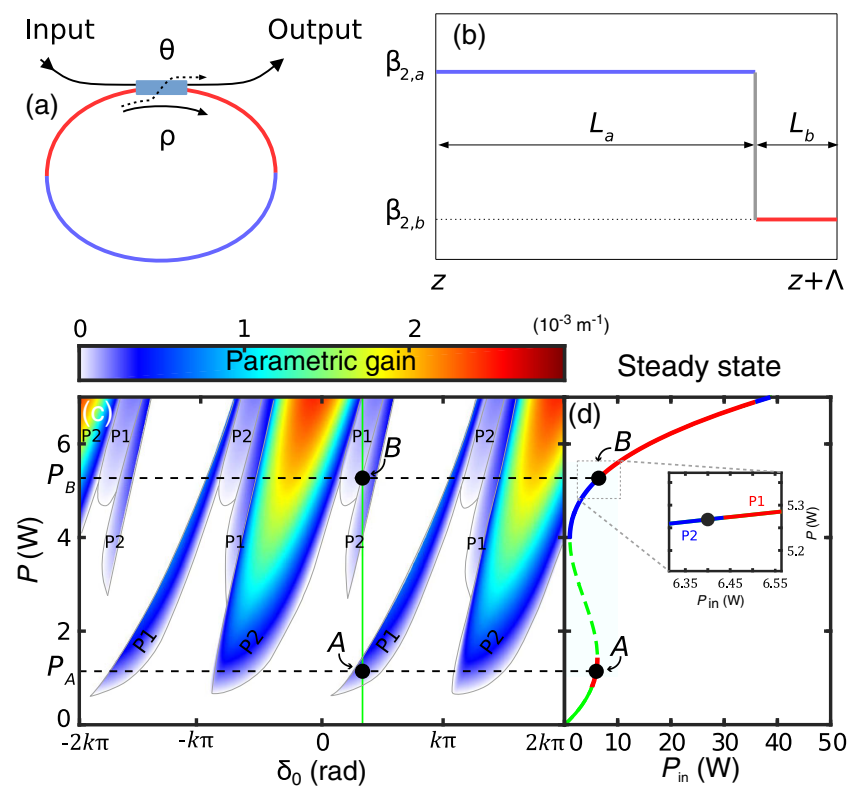

FIG. 2. (a) Schematic of the cavity. (b) GVD evolution over one cavity round trip. (c) 2D map of the maximum parametric gain in the plan $\left(\delta_{0}, P\right)$. (d) Steady-state curve of the cavity for $\delta_{0}=$ $1.1 \mathrm{rad}$ (green curve). The red (blue) parts stand for the region where the $\mathrm{P} 1(\mathrm{P} 2)$ regime can be excited. The inset zooms in on point $B$. Parameters are as follows: $\beta_{2}^{\mathrm{SMF}-28}=-19 \mathrm{ps}^{2} \cdot \mathrm{km}^{-1}$, $L^{\mathrm{SMF}-28}=1.6 \mathrm{~m}, \quad \beta_{2}^{\mathrm{DSF}}=2 \mathrm{ps}^{2} \cdot \mathrm{km}^{-1}, \quad L^{\mathrm{DSF}}=48.1 \mathrm{~m}, \quad \gamma=$ $5.5 \mathrm{~W}^{-1} \cdot \mathrm{km}^{-1}$, cavity losses $\alpha=0.157, L=\Lambda$.

with

$$
\begin{gathered}
\sigma_{1}=\frac{\left(\beta_{2, a} \mu_{a}\right)^{2}+\left(\beta_{2, b} \mu_{b}\right)^{2}}{2 \beta_{2, a} \beta_{2, b} \mu_{a} \mu_{b}}, \\
\sigma_{2 / 3}=\frac{\left(\beta_{2, a / b} \Omega^{2}\right)^{2}+4 \mu_{a / b}^{2}}{4 \mu_{a / b} \beta_{2, a / b} \Omega^{2}} .
\end{gathered}
$$

The parameter $\mu_{a / b}=\sqrt{\left(\beta_{2, a / b}^{2} \Omega^{4}\right) / 4+\beta_{2, a / b} \gamma P \Omega^{2}}$ refers to the standard MI gain, with $\Omega$ the pulsation of the perturbation for fibers $a$ and $b$. Unstable eigenvalues appear for the same condition as in uniform cavities, and again, they are always real. Consequently, introducing $\lambda^{\prime}$, which corresponds to the eigenvalue $\lambda_{ \pm}$with the highest modulus, the complex gain over a cavity round trip (i.e., over $N$ periods $\Lambda$ ) reads as $N \ln \left(\left|\lambda^{\prime}\right|\right) / \Lambda+i N m \pi / \Lambda$, with $m$ integer. From this expression, contrary to the case of the uniform cavity studied in Sec. II B, P1/P2 regimes are not only associated with the order $m$ but also with the number of dispersion periods forming the cavity $N$. Indeed, for $m$ odd and an odd number of dispersion periods, perturbations experience an exponential growth with a growth rate $g_{\mathrm{DMF}}=N \ln \left(\left|\lambda^{\prime}\right|\right) / \Lambda$ and a $\pi$ phase shift ( $\mathrm{P} 2$ regime) at each round trip, while in all other cases, there is no $\pi$ phase shift (P1 regime). A summary of all the combinations $(N, m)$ is presented in Table I. 
TABLE I. Conditions under which parametric instabilities exhibit P1 or P2 dynamics in dispersion-modulated cavities.

\begin{tabular}{|c|c|c|c|}
\hline & & \multicolumn{2}{|c|}{$N=L / \Lambda$} \\
\hline & & Even & Odd \\
\hline \multirow[t]{2}{*}{$m$} & Even & \multicolumn{2}{|c|}{ P1 } \\
\hline & Odd & P1 & P2 \\
\hline
\end{tabular}

For the sake of simplicity, we focus on the case where $N=1$, which is the easiest configuration to implement experimentally $[19,21,27,47,48]$.

Similarly to the uniform cavity studied in Sec. II B, Fig. 2(c) showcases a 2D map in the $\left(\delta_{0}, P\right)$ plane of the maximum gain value calculated from the growth rate $g_{\mathrm{DMF}}$. Many parametric instability tongues appear, characterized either by $\mathrm{P} 1$ or $\mathrm{P} 2$ dynamics. We work in a range of the detuning that allows us to observe Turing and Faraday instabilities, that is to say, P1 and P2 dynamics, respectively, by simply tuning the pump power, keeping a constant cavity detuning $[19,21,27,47]$. The cavity detuning is set to $\delta_{0}=1.1 \mathrm{rad}$ [marked by a vertical green line in Fig. 2(c)], which leads to a normalized cavity detuning $\Delta=7$ large enough to allow the Turing instability to reach a steady state [44]. By setting the pump power above the cavity threshold $\left(P_{A}=1.14 \mathrm{~W}\right.$, point $\left.A\right)$, the Turing instability is excited on the lower branch of the steadystate curve [Fig. 2(d)] and is characterized by P1 dynamics. The upper branch can be reached through an increase of the pump power up to $P=5.27 \mathrm{~W}$ (point $B$ ). The system then switches to another instability tongue, which is characterized by P2 dynamics [see inset in Fig. 2(d)]. The Faraday mechanism is known to be at the origin of multiple tongues of instability [49], and a further increase of the pump power would bring the system to a regime where another parametric instability tongue can be excited, characterized by P1 dynamics. As expected from previous theoretical works, the Turing instability is always ruled by a P1-type instability, while the Faraday one can be either $\mathrm{P} 1$ or $\mathrm{P} 2$.

\section{EXPERIMENTAL INVESTIGATIONS}

In this section, we experimentally study the emergence of $\mathrm{P} 1$ and $\mathrm{P} 2$ regimes in the temporal domain in uniform and dispersion-modulated cavities.

\section{A. Experimental setup}

The experimental setup is depicted in Fig. 3. It is similar to those used in Refs. [12,21]. It consists of a passive fiber cavity, made of either a uniform or a dispersion-modulated fiber (see parameters listed in Figs. 1 and 2) closed by a $90 / 10$ coupler. In the uniform cavity, the coupler is made of the same fiber as the whole cavity (a specially designed dispersion shifted fiber, $\beta_{2}^{\mathrm{DSF}}=9 \mathrm{ps}^{2} / \mathrm{km}$ ) to get a perfectly uniform cavity, while for the dispersion-modulated

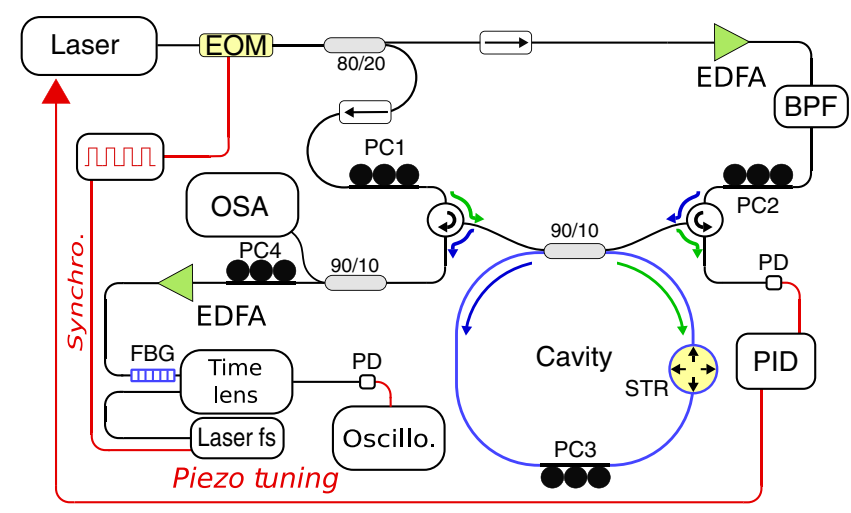

FIG. 3. Experimental setup. PC, polarization controller; STR, stretcher; PD, photodetector; EOM, electro-optic modulator; OSA, optical spectrum analyzer; EDFA, erbium-doped fiber amplifier; BPF, band-pass filter; PID, proportional-integralderivate controller; Laser fs, femtosecond laser; Oscillo., oscilloscope; FBG, fiber Bragg grating.

cavity, it is a standard SMF-28 fiber. To drive the cavity, a train of square pulses of 1-ns duration is used. It prevents Brillouin scattering and allows us to obtain high peak power to trigger the MI process. These pulses are generated from a cw laser at $1550.5 \mathrm{~nm}$ chopped by an electro-optical modulator (EOM). The repetition rate is set to match with the repetition rate of the cavity to get one pulse per round trip. Pulses are then amplified by an erbium-doped fiber amplifier (EDFA), and they pass through a thin filter (BPF, $1 \mathrm{~nm}$ width) to remove the excess amplified spontaneous emission (ASE). Pump pulses are launched into the cavity in the anticlockwise (blue arrows) direction, while a fraction of the output power of the EOM is launched in the clockwise direction (green arrows). This sample is used to stabilize the cavity to small external perturbations thanks to a feedback loop system (proportional-integral-derivativecontroller, which finely tunes the cw laser wavelength) $[11,19,26]$. The cavity output is studied by means of an optical spectrum analyzer (OSA) and a commercial time lens system (Picoluz ultrafast temporal magnifier, Thorlabs) based on the results published in Ref. [37]. The time lens system is pumped by a femtosecond laser centered at $1570 \mathrm{~nm}$. This laser will serve as a reference clock for the rest of the setup to get a perfect synchronization between all laser pulses involved in the experimental system. The cavity length is carefully adjusted with a fiber stretcher so that the laser repetition rate of the time lens pump, about $100 \mathrm{MHz}$, is an exact multiple of the cavity repetition rate (typically 59 times for the uniform cavity and 24 times for the modulated cavity). The magnified signal (magnified factor of 57) is recorded by a fast oscilloscope and photodiode (70-GHz bandpass each). However, we added a fiber Bragg grating (FBG), used in transmission and placed just before the time lens in order to lower the power of the central component. It allows us to reduce the relative amplitude of the cw component compared to the 
modulated ones in the time domain, in order to record the periodic temporal patterns. Thanks to this method, we recorded the temporal waveforms with approximately $300 \mathrm{fs}$ of resolution over a window of $50 \mathrm{ps}$. This resolution is short enough to record MI temporal patterns, whose period of modulation lies in the picosecond range.

\section{B. Experimental results}

Experimental results have been performed in both uniform and modulated cavities to observe the P1-P2 transition described in theory and marked by points $A, B$, and $C$ in Figs. 1(c) and Fig. 1(d) and by points A and B in Figs. 2(c) and 2(d). They have been compared with numerical simulations performed by integrating the Ikeda map model [Eqs. (1) and (2)]. Numerical simulations have been carried out using a square pulse pump of $1 \mathrm{~ns}$ as in experiments, adding a low random noise. In the case of Turing instabilities (lower branch of the bistable cycle), we used a cw pump seeded by a monochromatic signal located at the maximum gain frequency. For such large normalized cavity detuning values, the area of instability on the lower branch is very restricted and thus very sensitive to perturbations. Consequently, seeding the system with noise should lead to unexpected switches over the upper branch and avoid the observation of stable temporal patterns over a large round-trip number (typically 1000 in numerical simulations). In experiments, for the sake of clarity, we restricted our results to eight round trips, but we have been able to record this steady state over more than 30 round trips.

\section{Uniform cavity}

All experimental parameters are listed in the caption of Fig. 1. We investigate the configurations corresponding to points $A, B$, and $C$ in Fig. 1(c). First, we start by working on positive detuning to reach the first $\mathrm{P} 1$ regime tongue on Fig. 1 by increasing the input power. It corresponds to the configuration marked by point $A$ in Fig. 1(c) $\left(\delta_{0}=1.44 \mathrm{rad}\right.$ and $P=1.56 \mathrm{~W}$ ). Two weak sidebands, symmetric around the pump, are destabilized, as can be seen in Fig. 4(a). They are located at $140 \mathrm{GHz}$, in really good agreement with theory, predicting $154 \mathrm{GHz}$ [calculated from Eq. (3) and marked by vertical dashed lines in Fig. 4(a)]. The temporal pattern recorded for eight consecutive round trips is shown in a 2D color plot in Fig. 4(c), where the minimum and the maximum are normalized to 0 and 1 , respectively. We note that this pulse train identically reproduces itself round trip to round trip. The last two consecutive round-trip traces depicted in Fig. 4(b) (orange lines) show a good overlap. Thus, this result proves that the system operates in the P1 regime, as expected from theory. These results are confirmed by numerical simulations, with an excellent agreement for the spectrum displayed in Fig. 4(a) (blue lines) as well as for temporal traces depicted in Figs. 4(d)-4(b) corresponding, respectively, to the $2 \mathrm{D}$ color plot from intracavity temporal signals and the last two consecutive round-trip traces from (d). For the sake of clarity, we use the same normalization as in experiments.

To reach point $B$ of Fig. 1(c), we increase the input power until switching on the upper branch, where the system also features an instability zone [Fig. 1(d)]. First, we
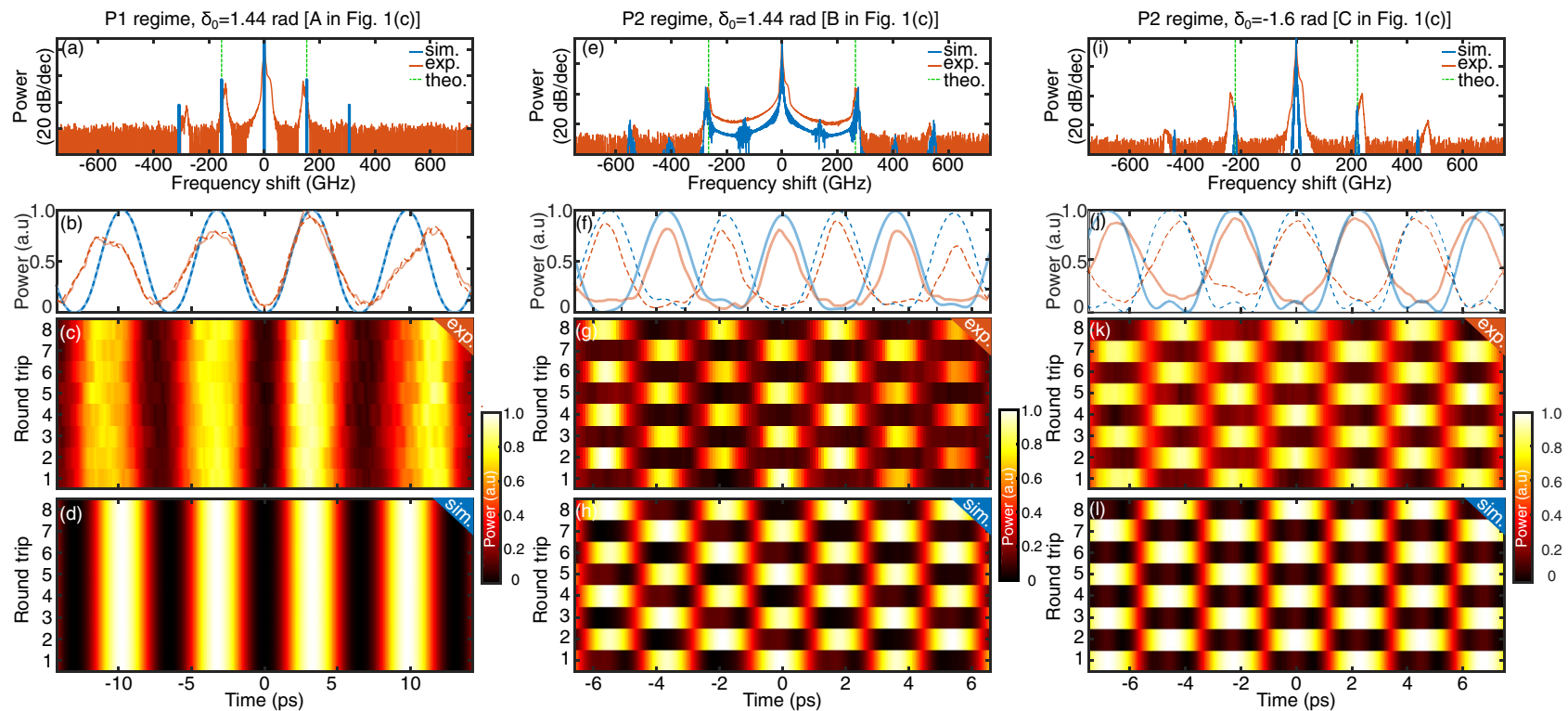

FIG. 4. (a)-(d) Related to point $A$, (e)-(h) to point $B$, and (i)-(l) to point $C$ in Fig. 1(c). (a,e,i) Output spectra, from experiment (orange lines) and simulations (blue lines). The green dashed vertical lines are the theoretical sideband positions. (b,f,j) Last two consecutive traces from experiment (orange lines) and simulation (blue lines). The 2D color plot shows temporal traces for eight consecutive round trips in experiments $(\mathrm{c}, \mathrm{g}, \mathrm{k})$ and in numerics $(\mathrm{d}, \mathrm{h}, \mathrm{l})$. Cavity parameters are listed in the caption of Fig. 1. 
observe a chaotic temporal pattern in good agreement with numerical simulation predictions (not shown here). Then, we slowly decrease it until the intracavity power reaches $P=5.84 \mathrm{~W}$ (input power of $6.0 \mathrm{~W}$ ) where the temporal waveform becomes periodic again. This method is the usual way to reach operating points located close to the knee of the steady-state curve of the upper branch. Results are depicted in Figs. 4(e)-4(h), similarly to the previous case. Two symmetric sidebands appear around the pump in the output spectrum at $273 \mathrm{GHz}$, in excellent agreement with theoretical predictions $[265 \mathrm{GHz}$ calculated from Eq. (3) and marked by vertical dashed lines in Fig. 4(e)] and with numerical simulations (blue lines). The normalized temporal traces obtained here alternate between two $\pi$ out-of-phase modulated patterns at each round trip with a singular form of a chessboard [Fig. 4(g)], which is also clear by looking at the last two round trips depicted in Fig. 4(f) (orange lines). The system undergoes the P2 regime as predicted by theory. Experimental results are in excellent agreement with numerical simulations [blue lines in Figs. 4(e) and 4(f), and in Fig. 4(h), corresponding to the 2D color plot from intracavity temporal signals] in this example, too. Finally, we change the cavity detuning to a negative value $\delta_{0}=-1.6 \mathrm{rad}$, to reach the first $\mathrm{P} 2$ regime tongue by increasing the input power [marked by point $C$ in Figs. 1(c) and 1(d)]. The cavity threshold is about 2 times larger in that case $\left(P_{\text {in }}=16.3 \mathrm{~W}\right.$, respectively $P=0.83 \mathrm{~W}$ ) compared to positive detuning cases investigated previously. Results are shown in Figs. 4(i)-4(1). Two symmetric sidebands appear around the pump at
$238 \mathrm{GHz}$, in excellent agreement with theoretical predictions $[221 \mathrm{GHz}$ according to theoretical predictions using Eq. (3) and marked by vertical dashed lines in Fig. 4(i)] and with numerical simulations (blue lines). The chessboard pattern in Fig. 4(k) clearly proves that the system undergoes a P2 regime, as expected from theory and in good agreement with numerical simulations [Fig. 4(1) and blue lines in Figs. 4(i) and 4(j)].

As in the positive detuning case, we could increase the input power to switch the system in another instability area where the temporal waveform undergoes a P1 regime, but this larger amount of power cannot be reached with our experimental system.

\section{Dispersion-modulated fiber cavities}

We now investigate the dispersion-modulated cavity whose parameters are listed in the caption of Fig. 2. The average dispersion is positive, so the system is known to be modulationaly unstable if it operates in the bistable regime of the LLE model. The turing instability only depends on the average dispersion value and can be observed on the lower branch, provided that the normalized detuning is larger than 4.25 [50]. The Faraday instability originates from the periodic variation of the dispersion and exists on the upper branch of the system $[19,27]$. We choose to study the simplest configuration for which the period of modulation of the dispersion is equal to the cavity length $(N=1)$. Except for the cavity, the experimental setup is identical to the one used in the previous section to investigate the
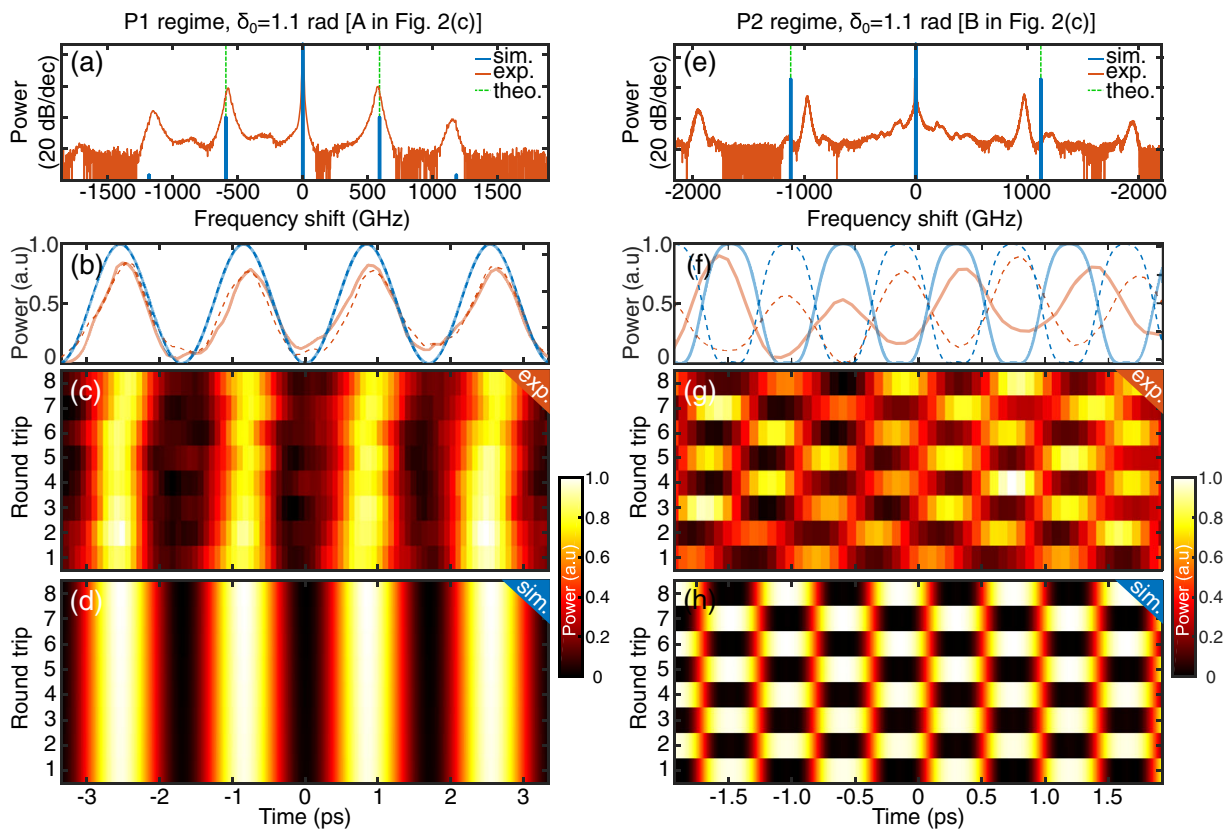

FIG. 5. (a)-(d) Related to point $A$ and (e)-(h) to point $B$ in Fig. 2(c). (a,e) Output spectra, from experiment (orange lines) and simulations (blue lines). The green dashed vertical lines are the theoretical sideband positions. (b,f) Last two consecutive traces from experiment (orange lines) and simulation (blue lines). The 2D color plot of temporal traces for eight consecutive round trips in experiments $(\mathrm{c}, \mathrm{g})$ and numerical simulations $(\mathrm{d}, \mathrm{h})$. Parameters are listed in the caption of Fig. 2. 
uniform cavity. We note that in dispersion-modulated cavities, the $\mathrm{P} 1$ and $\mathrm{P} 2$ regimes are, respectively, associated with Turing and Faraday instabilities, as has been shown theoretically [20]. Turing and Faraday instabilities are easily discriminated in the frequency domain $[19,21,27]$ and thus in the P1 and P2 regimes. However, the analysis does not provide a direct observation of these regimes. Here, our goal is to perform a direct observation in the time domain and to check experimentally that $\mathrm{P} 1$ is associated with Turing and P2 with Faraday instabilities.

We set the cavity detuning to $\delta_{0}=1.1 \mathrm{rad}(\Delta=7)$ to observe both regimes. Just above the Turing instability threshold $\left(P_{\text {in }}=5.95 \mathrm{~W}, P=1.14 \mathrm{~W}\right)$, two symmetrical sidebands around the pump are generated [see Fig. 5(a)]. They are located at $580 \mathrm{GHz}$, in really good agreement with theory, predicting $591 \mathrm{GHz}$ [calculated from Eq. (3) and marked by vertical dashed lines in Fig. 5(a)]. Increasing the input power up to $6.42 \mathrm{~W}(P=5.27 \mathrm{~W})$, the system switches onto the upper branch of the steady-state cycle, and thus the Faraday instability is excited. As expected from theory [18], their spectral positions are different from the Turing ones. We measure $975 \mathrm{GHz}$ in experiments, which is slightly different from theory, giving $1.122 \mathrm{THz}$ [from Eq. (3); see vertical dashed lines in Fig. 5(e)]. This discrepancy can be explained by the sensitivity of sideband frequencies to experimental parameters in this instability domain, particularly to the intracavity power, which is indirectly measured from the input power. Numerical simulations, represented by blue lines in Fig. 5(a) for the Turing instability, are in good agreement with experimental recordings (orange lines), while Fig. 5(e), for the Faraday instability, shows this small disagreement. Experimental temporal traces are shown in Figs. 5(b) and 5(c) and Figs. 5(f) and 5(g), respectively, for the Turing and Faraday instabilities, where the corresponding numerical simulations [Figs. 5(b) (blue lines) and 5(d) for Turing instabilities and Figs. 5(f) (blue lines) and 5(h) for Faraday instabilities] show the same behavior. From these figures, it is clear that the Turing instability lies in the P1 regime and the Faraday instability in the $\mathrm{P} 2$ regime, which is in excellent agreement with numerical simulations.

\section{CONCLUSION}

Real-time characterization with a resolution of the order of hundreds of femtoseconds allowed by time lens systems revolutionizes the way we investigate passive resonators for cavity soliton characterization [4] or lasers for real-time characterization of mode-locking dynamics [40] in phase and intensity. In this work, we show that it can also be of great interest in the context of modulation instability. We anticipate that these time lens systems or, more recently, dual-frequency comb systems [51] will become a standard in future experiments to get real-time recordings with high resolution to improve the understanding of the complex dynamics occurring in these nonlinear systems.

\section{ACKNOWLEDGMENTS}

This work was partly supported by the Agence Nationale de la Recherche through the Labex Centre Europeen pour les Mathematiques, la Physique et leurs Interactions (CEMPI), and Equipex Fibres optiques pour les hauts flux (FLUX) through the "Programme Investissements d'Avenir," by the Ministry of Higher Education and Research, Hauts de France Council and European Regional Development Fund (ERDF) through the Contrat de Projets Etat-Region (CPER Photonics for Society, P4S) and HEAFISY project and SITE ULNE through the FUHNKC project.

[1] T. J. Kippenberg, A. L. Gaeta, M. Lipson, and M. L. Gorodetsky, Dissipative Kerr Solitons in Optical Microresonators, Science 361, eaan8083 (2018).

[2] A. Pasquazi, M. Peccianti, L. Razzari, D. J. Moss, S. Coen, M. Erkintalo, Y. K. Chembo, T. Hansson, S. Wabnitz, P. Del'Haye, X. Xue, A. M. Weiner, and R. Morandotti, Micro-Combs: A Novel Generation of Optical Sources, Phys. Rep. 729, 1 (2018).

[3] F. Leo, S. Coen, P. Kockaert, S.-P. Gorza, P. Emplit, and M. Haelterman, Temporal Cavity Solitons in One-Dimensional Kerr Media as Bits in an All-Optical Buffer, Nat. Photonics 4, 471 (2010).

[4] T. Herr, V. Brasch, J. D. Jost, C. Y. Wang, N. M. Kondratiev, M. L. Gorodetsky, and T. J. Kippenberg, Temporal Solitons in Optical Microresonators, Nat. Photonics 8, 145 (2014).

[5] L. A. Lugiato and R. Lefever, Spatial Dissipative Structures in Passive Optical Systems, Phys. Rev. Lett. 58, 2209 (1987).

[6] M. Haelterman, S. Trillo, and S. Wabnitz, Dissipative Modulation Instability in a Nonlinear Dispersive Ring Cavity, Opt. Commun. 79, 401 (1997).

[7] P. Del'Haye, T. Herr, E. Gavartin, M. L. Gorodetsky, R. Holzwarth, and T. J. Kippenberg, Octave Spanning Tunable Frequency Comb from a Microresonator, Phys. Rev. Lett. 107, 063901 (2011).

[8] T. Hansson, D. Modotto, and S. Wabnitz, Dynamics of the Modulational Instability in Microresonator Frequency Combs, Phys. Rev. A 88, 023819 (2013).

[9] S. Coen and M. Haelterman, Modulational Instability Induced by Cavity Boundary Conditions in a Normally Dispersive Optical Fiber, Phys. Rev. Lett. 79, 4139 (1997).

[10] F. Leo, A. Mussot, P. Kockaert, P. Emplit, M. Haelterman, and M. Taki, Nonlinear Symmetry Breaking Induced by Third-Order Dispersion in Optical Fiber Cavities, Phys. Rev. Lett. 110, 104103 (2013).

[11] A. Mussot, E. Louvergneaux, N. Akhmediev, F. Reynaud, L. Delage, and M. Taki, Optical Fiber Systems Are Convectively Unstable, Phys. Rev. Lett. 101, 113904 (2008).

[12] F. Bessin, F. Copie, M. Conforti, A. Kudlinski, and A. Mussot, Modulation Instability in the Weak Normal Dispersion Region of Passive Fiber Ring Cavities, Opt. Lett. 42, 3730 (2017).

[13] M. Anderson, Y. Wang, F. Leo, S. Coen, M. Erkintalo, and S. G. Murdoch, Coexistence of Multiple Nonlinear States in 
a Tristable Passive Kerr Resonator, Phys. Rev. X 7, 031031 (2017).

[14] T. Hansson and S. Wabnitz, Frequency Comb Generation Beyond the Lugiato-Lefever Equation: Multi-Stability and Super Cavity Solitons, J. Opt. Soc. Am. B 32, 1259 (2015).

[15] M. Conforti and F. Biancalana, Multi-Resonant LugiatoLefever Model, Opt. Lett. 42, 3666 (2017).

[16] T. Hansson, M. Bernard, and S. Wabnitz, Modulational Instability of Nonlinear Polarization Mode Coupling in Microresonators, J. Opt. Soc. Am. B 35, 835 (2018).

[17] J. Fatome, B. Kibler, F. Leo, A. Bendahmane, G.-L Oppo, B. Garbin, Y. Wang, S. G. Murdoch, M. Erkintalo, and S. Coen, in Advanced Photonics 2018 (BGPP, IPR, NP, NOMA, Sensors, Networks, SPPCom, SOF) (2018), Paper NpTu4C.6 (Optical Society of America, 2018), p. NpTu4C.6.

[18] M. Conforti, A. Mussot, A. Kudlinski, and S. Trillo, Modulational Instability in Dispersion Oscillating Fiber Ring Cavities, Opt. Lett. 39, 4200 (2014).

[19] F. Copie, M. Conforti, A. Kudlinski, A. Mussot, and S. Trillo, Competing Turing and Faraday Instabilities in Longitudinally Modulated Passive Resonators, Phys. Rev. Lett. 116, 143901 (2016).

[20] M. Conforti, F. Copie, A. Mussot, A. Kudlinski, and S. Trillo, Parametric Instabilities in Modulated Fiber Ring Cavities, Opt. Lett. 41, 5027 (2016).

[21] F. Copie, M. Conforti, A. Kudlinski, S. Trillo, and A. Mussot, Modulation Instability in the Weak Dispersion Regime of a Dispersion Modulated Passive Fiber-Ring Cavity, Opt. Express 25, 11283 (2017).

[22] K. Staliunas, C. Hang, and V. V. Konotop, Parametric Patterns in Optical Fiber Ring Nonlinear Resonators, Phys. Rev. A 88, 023846 (2013).

[23] M. Haelterman, Simple Model for the Study of PeriodDoubling Instabilities in the Nonlinear Ring Cavity, Appl. Phys. Lett. 61, 2756 (1992).

[24] A. Ankiewicz and C. Pask, Chaos in Optics: Field Fluctuations for a Nonlinear Optical Fibre Loop Closed by a Coupler, J. Austral. Soc. Ser. B 29, 1 (1987).

[25] M. Haelterman, Ikeda Instability and Transverse Effects in Nonlinear Ring Resonators, Opt. Commun. 100, 389 (1993).

[26] S. Coen, M. Haelterman, P. Emplit, L. Delage, L. M. Simohamed, and F. Reynaud, Experimental Investigation of the Dynamics of a Stabilized Nonlinear Fiber Ring Resonator, JOSA B 15, 2283 (1998).

[27] F. Copie, M. Conforti, A. Kudlinski, S. Trillo, and A. Mussot, Dynamics of Turing and Faraday Instabilities in a Longitudinally Modulated Fiber-Ring Cavity, Opt. Lett. 42, 435 (2017).

[28] K. Ikeda and O. Akimoto, Instability Leading to Periodic and Chaotic Self-Pulsations in a Bistable Optical Cavity, Phys. Rev. Lett. 48, 617 (1982).

[29] G. Steinmeyer, D. Jaspert, and F. Mitschke, Observation of a Period-Doubling Sequence in a Nonlinear Optical Fiber Ring Cavity Near Zero Dispersion, Opt. Commun. 104, 379 (1994).

[30] M. Nakazawa, K. Suzuki, and H. A. Haus, Modulational Instability Oscillation in Nonlinear Dispersive Ring Cavity, Phys. Rev. A 38, 5193 (1988).
[31] R. Vallée, Temporal Instabilities in the Output of an All-Fiber Ring Cavity, Opt. Commun. 81, 419 (1991).

[32] N. Akhmediev, J. M. Soto-Crespo, and G. Town, Pulsating Solitons, Chaotic Solitons, Period Doubling, and Pulse Coexistence in Mode-Locked Lasers: Complex GinzburgLandau Equation Approach, Phys. Rev. E 63, 056602 (2001).

[33] S. Boscolo, S. K. Turitsyn, and C. Finot, Amplifier Similariton Fiber Laser with Nonlinear Spectral Compression, Opt. Lett. 37, 4531 (2012).

[34] L. Feng, P. K. A. Wai, and J. N. Kutz, Geometrical Description of the Onset of Multi-Pulsing in ModeLocked Laser Cavities, J. Opt. Soc. Am. B, 27, 2068 (2010).

[35] L. Luo, T. J. Tee, and P. L. Chu, Chaotic Behavior in Erbium-Doped Fiber-Ring Lasers, J. Opt. Soc. Am. B 15, 972 (1998).

[36] G. Sucha, S. R. Bolton, S. Weiss, and D. S. Chemla, Period Doubling and Quasi-Periodicity in Additive-Pulse Mode-Locked Lasers, Opt. Lett. 20, 1794 (1995).

[37] R. Salem, M. A. Foster, and A. L. Gaeta, Application of Space-Time Duality to Ultrahigh-Speed Optical Signal Processing, Adv. Opt. Photonics 5, 274 (2013).

[38] P. Suret, R. E. Koussaifi, A. Alexey, C. Evain, S. Randoux, S. Szwaj, and S. Bielawski, Single-Shot Observation of Optical Rogue Waves in Integrable Turbulence Using Time Microscopy, Nat. Commun. 7, 13136 (2016).

[39] M. Närhi, B. Wetzel, C. Billet, S. Toenger, T. Sylvestre, J.-M Merolla, R. Morandotti, F. Dias, G. Genty, and J.M. Dudlet, Real-Time Measurements of Spontaneous Breathers and Rogue Wave Events in Optical Fibre Modulation Instability, Nat. Commun. 7, 13675 (2016).

[40] P. Ryczkowski, M. Närhi, C. Billet, J.-M. Merolla, G. Genty, and J. M. Dudley, Real-Time Full-Field Characterization of Transient Dissipative Soliton Dynamics in a Mode-Locked Laser, Nat. Photonics 12, 221 (2018).

[41] B. H. Kolner and M. Nazarathy, Temporal Imaging with a Time Lens, Opt. Lett. 14, 630 (1989).

[42] D. W. McLaughlin, J. V. Moloney, and A. C. Newell, New Class of Instabilities in Passive Optical Cavities, Phys. Rev. Lett. 54, 681 (1985).

[43] S. Coen and M. Haelterman, Competition between Modulational Instability and Switching in Optical Bistability, Opt. Lett. 24, 80 (1999).

[44] S. Coen, M. Haelterman, P. Emplit, L. Delage, L. M. Simohamed, and F. Reynaud, Bistable Switching Induced by Modulational Instability in a Normally Dispersive All-Fibre Ring Cavity, J. Opt. B 1, 36 (1999).

[45] A. Mussot, M. Conforti, S. Trillo, F. Copie, and A. Kudlinski, Modulation Instability in Dispersion Oscillating Fibers, Adv. Opt. Photonics 10, 1 (2018).

[46] N. Tarasov, A. M. Perego, D. V. Churkin, K. Staliunas, and S. K. Turitsyn, Mode-Locking via Dissipative Faraday Instability, Nat. Commun. 7, 12441 (2016).

[47] F. Copie, M. Conforti, A. Kudlinski, A. Mussot, F. Biancalana, and S. Trillo, Instabilities in Passive Dispersion Oscillating Fiber Ring Cavities, Europhys. J. D 71, 133 (2017). 
[48] S.-W. Huang, A. K. Vinod, J. Yang, M. Yu, D.-L. Kwong, and C. W. Wong, Quasi-Phase-Matched Multispectral Kerr Frequency Comb, Opt. Lett. 42, 2110 (2017).

[49] A. L. Lin, M. Bertram, K. Martinez, H. L. Swinney, A. Ardelea, and G. F. Carey, Resonant Phase Patterns in a Reaction-Diffusion System, Phys. Rev. Lett. 84, 4240 (2000).
[50] S. Coen and M. Haelterman, Continuous-Wave Ultrahigh-Repetition-Rate Pulse-Train Generation through Modulational Instability in a Passive Fiber Cavity, Opt. Lett. 26, 39 (2001).

[51] X. Yi, Q.-F. Yang, K. Y. Yang, and K. Vahala, Imaging Soliton Dynamics in Optical Microcavities, Nat. Commun. 9, 3565 (2018). 